\title{
MODELO DE AVALIAÇÃO DE DESEMPENHO GLOBAL
}

\section{GLOBAL PERFORMANCE EVALUATION MODEL}

\section{DOI: http://dx.doi.org/10.21714/raunp.v10i1.1513}

\section{Rolf Hermann Erdmann}

Doutor em Engenharia de Produção. Professor Titular da Universidade Federal de Santa Catarina.

E-mail: rolf.erdmann@ufsc.br

\section{Thiago Coelho Soares}

Doutor em Administração. Professor da Universidade do Sul de Santa Catarina - Unisul.

E-mail: tcoelhos@hotmail.com

Envio em: Junho de 2016

Aceite em: Fevereiro de 2018

\section{RESUMO}

0 objetivo deste artigo consiste em como um modelo de avaliação global pode gerar práticas de gestão institucional. Para isto utilizou-se como base quatro modelos de avaliação de desempenho: Avaliação institucional como sendo as avaliações realizadas pelo MEC (1) Avaliação institucional externa do MEC, (2) Avaliação de curso do MEC, (3) Avaliação de programa de pós-graduação CAPES, (4) Avaliação baseada em fatores de competitividade. Ao final, destes quatro modelos se elaborará um modelo de avaliação global. A análise foi feita comparando os quatro modelos apresentados na fundamentação teórica deste artigo. Para a comparação entre os indicadores do modelo utilizaram-se os princípios de análise da grounded theory. Assim, procedeu-se a codificação aberta, a codificação axial e a codificação seletiva. Desta forma, na codificação aberta se identificou os conceitos dos indicadores pertencentes aos quatro modelos. Na codificação axial se agrupou os indicadores em torno dos subsistemas produtivos de acordo com a sua semelhança conceitual. Por fim, na codificação seletiva, se atinge a categorização final e apresenta-se 0 agrupamento definitivo. Com 0 modelo gerado é possível avaliar com base em fatores de competitividade por meio de aplicação em sistemas de produção. Como o modelo de avaliação baseada em fatores de competitividade indica a geração de melhores práticas administrativas, conclui-se que este estudo promoveu reflexões e contribuições para a melhoria do desempenho das organizações educacionais e para 0 aprofundamento de estudos de novos modelos.

Palavras-chave: Avaliação Institucional. Avaliação de Desempenho. Fatores de Competitividade.

\section{ABSTRACT}

This paper aims to verify how the institutional review can generate management practices through a comprehensive evaluation model. Importantly, it is considered in this paper as institutional assessment and evaluations carried out by MEC (external institutional assessment and evaluation of progress) and CAPES (assessment of graduate program). The article was justified because it tries to improve the process of institutional review sought to generate a comprehensive evaluation model for HEI (Higher Education Institution), based on the assumption that a comprehensive evaluation model promotes advantages to the institution. The evaluation model, inspired by competitive factors, formed the basis for the other models, which have been incorporated by means of indicators. Thus, there is a generic model for the assessment of a production system, plus specific indicators of institutional evaluation, generating a global model for management of HEls. As the model based on competitive factors evaluation indicates the generation of best management practices, it is expected that the results of this study bring reflections and contributions to the improvement of the performance of educational organizations and to further studies of new models.

Keywords: Institutional Assessment. Management. Competitiveness Factors. 


\section{INTRODUÇÃO}

As Instituições de Ensino Superior (IES) exigem dos seus gestores criatividade na formulação de modelos e abordagens gerenciais que lhes permitam atuar em um contexto complexo caracterizado por objetivos difusos, tecnologia múltipla, liberdade acadêmica, natureza qualitativa do trabalho e grande sensibilidade a fatores ambientais. Para cumprir o seu trabalho, o gestor universitário necessita reunir não só competências técnicas e políticas, mas também habilidades de um artesão ao lidar com as expectativas, intenções, angústias, frustrações e necessidades das pessoas na organização (MEYER JUNIOR; MEYER; 2004). Da mesma forma que nos demais setores, os indicadores no ensino superior tendem a ser históricos e geralmente incapazes de alertar as instituições para as mudanças de forma tempestiva. Além disso, a ênfase tem sido em medidas acadêmicas mais fáceis de serem obtidas (RUBEN, 1999).

Verifica-se de forma geral, nas organizações, uma tendência em relação aos sistemas de avaliação de desempenho ficarem reduzidos à dimensão financeira. No ensino superior existe também esta tendência, contudo, sua ênfase está voltada as medidas acadêmicas em virtude de estarem mais próximas ao seu core business.

Na história recente do Brasil, no que diz respeito à avaliação da educação superior - dos anos de 1960 até os dias atuais - as iniciativas, no seu âmago, foram revestidas por questões ideológicas. Na concepção "tradicional" o conhecimento é entendido como um "produto" (DIAS SOBRINHO, 2004, p. 704-705). Portanto, devem ser orientadas pela eficiência e resultados lucrativos dos seus processos. E neste caso, o processo avaliativo, configura-se como forma de controle orientada pelo e para o mercado. No entanto, quando se entende que o conhecimento é um bem público de ordem social, a avaliação toma outro sentido. O processo avaliativo passa a buscar o melhoramento, a emancipação, a formação e a evolução de todos os agentes envolvidos, inclusive do próprio conhecimento como elemento singular do desenvolvimento social (LIMA et al, 2012).

Com um modelo de avaliação de desempenho global e único, as IES podem, conforme Conto, Britto Cyrne (2011) obter vantagens em custos, uma vez que evita a duplicidade de atividades e de estrutura administrativa; vantagens operacionais, pois a proposta de alinhamento organiza administrativamente a Institui- ção de modo que as atividades e operações sejam realizadas de maneira natural, sem a necessidade de implantação de novas estruturas; e vantagens legais, de modo que o modelo além de possibilitar melhoria na gestão da Instituição, também permite o cumprimento das exigências legais do Ministério da Educação.

Considerando o exposto, o objetivo deste artigo consiste em como um modelo de avaliação global pode gerar práticas de gestão institucional. Para isto, utilizou-se como base quatro modelos de avaliação de desempenho: Avaliação institucional como sendo as avaliações realizadas pelo MEC (1) Avaliação institucional externa do MEC, (2) Avaliação de curso do MEC, (3) Avaliação de programa de pós-graduação CAPES, (4) Avaliação baseada em fatores de competitividade.

$\mathrm{O}$ artigo se justifica porque tenta melhorar o processo de avaliação institucional procurando gerar um modelo de avaliação global para IES (Instituição de Ensino Superior), partindo do pressuposto de que um modelo de avaliação global promove vantagens para a instituição (GRZEBIELUCKAS et al, 2011; CONTO; BRITTO; CYRNE, 2011; GALVÃO; CORRÊA; ALVES, 2011; ENSSLIN et al, 2012; CRISPIM; LUGOBONI, 2012; LONGARAY; ENSSLIN, 2013).

\section{FUNDAMENTAÇÃO TEÓRICA}

A implementação de uma ferramenta de avaliação de desempenho leva a mudança e o aprendizado. Em caso de sucesso, a organização estará mais desenvolvida e em nível evolutivo superior, caracterizada pela aquisição de competências para lidar com a mudança (ROMAN et al, 2017). Assim, a fundamentação teórica deste capítulo abordará as avaliações legais aplicadas as IES: avaliação institucional e avaliação de curso aplicada pelo MEC e avaliação da pós-graduação aplicada pela CAPES. Além disto, será abordada a metodologia de avaliação de desempenho baseada em fatores de competitividade.

\subsection{AVALIAÇÃO INSTITUCIONAL}

De acordo com Peterson (2000), existe uma diversidade conceitual e metodológica relacionada à avaliação institucional, que está envolta em um complexo cenário institucional, em que convivem diferentes abordagens e dinâmicas. Esta diversidade se materializa em uma multiplicidade de definições, tipos ou níveis, finalidades, enfoques, apoiadores, modelos 
conceituais e padrões de desempenho. Para Dias Sobrinho; Ristoff (2002, p.40), "a avaliação é plurirreferencial, semanticamente plural, dotada, pois, de múltiplos sentidos e interesses distintos e contraditórios, como tudo que se realiza na esfera social e pública dos valores e ideologias".

O SINAES pode ser considerado como uma ação integradora das iniciativas anteriormente desarticuladas - regulação e avaliação - em um Sistema que soma os esforços das instituições com os esforços governamentais.

O SINAES é um programa de avaliação da educação superior que tem por finalidade permitir o acompanhamento da evolução da qualidade do ensino superior e permitir que o MEC conduza o processo de credenciamento ou renovação de credenciamento de instituições de educação superior e de autorização, reconhecimento e renovação de reconhecimento de cursos de graduação, em consonância com o Art. $9^{\circ}$, inciso IX, da Lei $n^{0}$ 9.394, de 1996. Sua coordenação se faz pela Comissão Nacional de Avaliação da Educação Superior (CONAES), órgão do âmbito do Ministério da Educação, vinculada ao Gabinete do Ministro de Estado (BRASIL, 2004).

A Lei 10.861 assegura que a avaliação das instituições deverá utilizar procedimentos e instrumentos diversificados, dentre os quais a auto-avaliação e a avaliação externa in loco. Terá por objetivo identificar perfil e o significado da atuação das instituições, por meio de suas atividades, cursos, programas, projetos e setores, considerando as suas diferentes dimensões, dentre elas obrigatoriamente as seguintes (BRASIL, 2004):

(a) a missão e o plano de desenvolvimento institucional;

(b) a política para o ensino, a pesquisa, a pós-graduação, a extensão e as respectivas formas de operacionalização, incluídos os procedimentos para estímulo à produção acadêmica, as bolsas de pesquisa, de monitoria e demais modalidades;

(c) a responsabilidade social da instituição, considerada especialmente no que se refere à sua contribuição em relação à inclusão social, ao desenvolvimento econômico e social, à defesa do meio ambiente, da memória cultural, da produção artística e do patrimônio cultural;

(d) a comunicação com a sociedade;

(e) as políticas de pessoal, as carreiras do corpo docente e do corpo técnico-administrativo, seu aperfeiçoamento, desenvolvimento profissional e suas condições de trabalho;

(f) organização e gestão da instituição, especialmen- te o funcionamento e representatividade dos colegiados, sua independência e autonomia na relação com a mantenedora, e a participação dos segmentos da comunidade universitária nos processos decisórios;

(g) infraestrutura física, especialmente a de ensino e de pesquisa, biblioteca, recursos de informação e comunicação;

(h) planejamento e avaliação, especialmente os processos, resultados e eficácia da auto-avaliação institucional;

(i) políticas de atendimento aos estudantes; e

(j) sustentabilidade financeira, tendo em vista o significado social da continuidade dos compromissos na oferta da educação superior

Após apresentada a avaliação institucional, na sequência será apresentada a avaliação do curso.

\subsection{AVALIAÇÃO DE CURSO}

A avaliação dos cursos de graduação, conforme Polidoro, Marinho-Araújo e Barreyro (2006, p. 432), é

\begin{abstract}
uma prática que já vinha sendo desenvolvida no sistema anterior; no entanto, para atender aos princípios do SINAES, adquiriu novas características, consistindo numa avaliação externa realizada por uma equipe multidisciplinar de especialistas para avaliar cursos de áreas afins, aos quais junta-se um avaliador institucional. Esse eixo terá sua articulação com os processos de regulação por estar definido na lei que os seus resultados estão vinculados com o reconhecimento e renovação (de reconhecimento) dos cursos.
\end{abstract}

O sistema de avaliação de cursos superiores no Brasil é conduzido pelo Instituto Nacional de Estudos e Pesquisas Educacionais Anísio Teixeira (Inep). Este instituto produz indicadores e mantém um sistema de informações que subsidia tanto o processo de regulamentação, exercido pelo Ministério da Educação (MEC), como também visa garantir transparência dos dados sobre a qualidade da educação superior à sociedade.

No âmbito do Sinaes e da regulação dos cursos de graduação no País, prevê-se que os cursos sejam avaliados periodicamente. Assim, os cursos de educação superior passam por três tipos de avaliação: para autorização, para reconhecimento e para renovação de reconhecimento.

De acordo com o Ministério da Educação (2012), a avaliação para renovação de reconhecimento é feita 
de acordo com o "ciclo do Sinaes", ou seja, a cada três anos. Nesta avaliação é calculado o Conceito Preliminar do Curso (CPC), um indicador prévio da situação dos cursos de graduação no país, o qual varia de 1 a 5. Os cursos que obtiverem conceito preliminar 1 ou 2 serão avaliados in loco por dois avaliadores ao longo de dois dias. Os cursos com conceito 3 e 4 receberão visitas apenas se solicitarem. Os cursos que obtiveram conceito 5 estão dispensados da avaliação in loco e terão os seus processos encaminhados à Secretaria Reguladora, para expedição da Portaria de renovação de reconhecimento.

Conforme Polidori, Marinho-Araújo e Barreyro (2006), outro aspecto importante é a capacitação dos especialistas, pois a intenção do sistema não é realizar ações de rastreamento, perseguição ou de punição, mas, sim, permitir que as Instituições de Ensino Superior possam, com o auxílio de especialistas, dinamizar as suas atividades, resolver questões prementes e construir metas concisas e pontuais. Para tal, as orientações dispensadas aos especialistas, devem ser de outra ordem além daquela que orienta a utilização de formulários eletrônicos e evidencia cumprimento de prazos. Apesar de estes pontos serem importantes, eles não podem ser os pontos centrais de um processo avaliativo que busca a melhoria da qualidade da educação superior oferecida no país.

O Sinaes é composto por três eixos que compreendem a avaliação das instituições, dos cursos e dos estudantes. De acordo com Polidori, Marinho-Araujo e Barreyro (2006), o primeiro eixo, que corresponde ao desenvolvimento da "avaliação das instituições", tem como principal objetivo verificar como as IES estão constituídas e qual a sua capacidade de atendimento à comunidade acadêmica. Neste eixo está incluído o desenvolvimento do processo de auto-avaliação coordenada pelas CPAs.

O segundo eixo compreende a avaliação dos "estudantes", cujo principal instrumento chama-se Exame Nacional de Desempenho dos Estudantes ENADE. Em relação ao Provão, o ENADE permite uma coleta mais rica de informações, possibilitando às IES a realização de debates e modificações nas suas questões acadêmicas com base em informações mais consistentes.

Já o terceiro eixo do Sinaes, de acordo com Ristoff e Giolo (2006) se refere à "avaliação dos cursos de graduação" consistido de uma avaliação externa realizada por uma equipe multidisciplinar de especialistas para avaliar cursos de áreas afins, aos quais se junta um avaliador institucional. Avaliam-se, sobretu- do, os conhecimentos e saberes necessários à formação das competências estabelecidas a partir do perfil do egresso: a) a estrutura e conteúdo curricular; b) o ementário, as bibliografias básica e complementar; c) as estratégias de ensino; d) a titulação dos docentes; e) os recursos materiais; f) os serviços administrativos; g) os serviços de laboratórios; h) a infraestrutura de apoio ao pleno funcionamento do curso.

A avaliação dos cursos é realizada com base em aspectos que possibilitam uma visão ampla e importante de diversos elementos que, conjuntamente, são referência para a qualidade dos cursos. A avaliação bem conduzida gera subsídios para o encaminhamento de ações para a manutenção ou melhorias em cada aspecto, convergindo para melhores níveis de performance dos cursos.

\subsection{AVALIAÇÃO DA PÓS-GRADUAÇÃO}

O Sistema de Avaliação da Pós-graduação foi implantado pela CAPES em 1976 e desde então vem cumprindo papel de fundamental importância para o desenvolvimento da pós-graduação e da pesquisa científica e tecnológica no Brasil, dando cumprimento aos seguintes objetivos, conforme a CAPES (2013):

- Estabelecer o padrão de qualidade exigido dos cursos de mestrado e de doutorado e identificar os cursos que atendem a tal padrão;

- Fundamentar, nos termos da legislação em vigor, os pareceres do Conselho Nacional de Educação sobre autorização, reconhecimento e renovação de reconhecimento dos cursos de mestrado e doutorado brasileiros - exigência legal para que estes possam expedir diplomas com validade nacional reconhecida pelo Ministério da Educação, MEC;

- Impulsionar a evolução de todo o Sistema Nacional de Pós-graduação, SNPG, e de cada programa em particular, antepondo-lhes metas e desafios que expressam os avanços da ciência e tecnologia na atualidade e o aumento da competência nacional nesse campo;

- Contribuir para o aprimoramento de cada programa de pós-graduação, assegurando-lhe o parecer criterioso de uma comissão de consultores sobre os pontos fracos e fortes de seu projeto e de seu desempenho e uma referência sobre o estágio de desenvolvimento em que se encontra;

- Contribuir para o aumento da eficiência dos programas no atendimento das necessidades nacionais e regionais de formação de recursos humanos de alto nível; 
- Dotar o país de um eficiente banco de dados sobre a situação e evolução da pós-graduação;

- Oferecer subsídios para a definição da política de desenvolvimento da pós-graduação e para a fundamentação de decisões sobre as ações de fomento dos órgãos governamentais na pesquisa e pós-graduação.

Maccari et al (2009) afirmam que o sistema de avaliação tem grande influência no desenvolvimento dos programas e é o seu principal direcionador estratégico, bem como os critérios e itens de avaliação estão bem definidos e são conhecidos pela comunidade acadêmica avaliada. Enfatizam que o sistema demanda alta qualidade e produtividade e encoraja os programas a ampliarem sua inserção na comunidade.

Silva (2009) analisa as características da produção docente submetida à avaliação dos Programas de Pós-Graduação e conclui que: a) o elemento chave da avaliação é o docente, sobretudo o do quadro permanente; b) os critérios de avaliação destacam a produção intelectual (que se refere às publicações qualificadas) e valorizam as publicações internacionais qualificadas; c) cresceram as atividades docentes de formação e publicações, tendendo a centralizar-se na Pós- Graduação; d) a condição do trabalho docente, de modo geral, reflete a política de avaliação dos Programas.

\subsection{AVALIÇÃO BASEADA EM FATORES DE COMPETITIVIDADE}

O contexto contemporâneo pode ser caracterizado pela incerteza gerada pela velocidade e intensidade das mudanças nas variáveis ambientais. Nesse sentido, a teoria da complexidade abordada por Luhmann (1997) e Morin (1982) tenta explicar essa nova realidade, que vai além da causalidade linear e da lógica formal, dominantes por um longo período, para um cenário de hipercomplexidade.

Ao utilizar a teoria da complexidade para o entendimento das organizações, é possível perceber as inter-relações existentes e, a partir desta percepção, pode-se atuar de maneira mais efetiva na obtenção de resultados (MOREIRA, 2011). Isto ocorre porque a organização é compreendida como um sistema integrado, sendo assim seu desempenho e nível de competitividade depende de diversas variáveis e da forma como elas se relacionam.

A análise dessas variáveis, de forma integrada, e a identificação de boas práticas é um caminho para ao compartilhar conhecimentos melhorar o desem- penho de outras organizações similares. $\mathrm{O}$ foco das boas práticas empresariais está na redução de desperdícios e no aumento da competitividade, por meio de uma resposta mais rápida e de menores custos (ROMAN et al, 2012). A evolução das pesquisas sobre boas práticas de gestão em organizações complexas aponta para 13 categorias de análise: Controle da Produção, Desempenho Operacional, Desenvolvimento de Novos Produtos, Fábrica/ Instalações, Gestão Ambiental, Investimentos, Organização e Cultura, Planejamento da Produção, Programação da Produção, Qualidade, Saúde e Segurança, Tecnologia, e Tempo de Ciclo (SILVEIRA, 2010).

Entende-se por categorias de análise, o agrupamento de conceitos que representam os pontos em que a gestão tem centrado seus esforços de aperfeiçoamento, baseando-se nos conceitos trazidos pelas boas práticas da gestão da produção. Ao subdividir o sistema em categorias de análise tem-se o aumento da complexidade, o que permite que o sistema consiga absorver e responder a complexidade do ambiente em que está inserida. Essas categorias são de tal forma interligadas que, ao agir em uma, as outras consequentemente serão afetadas (SILVEIRA, 2010).

O controle da produção é a categoria de análise de organizações complexas que visa garantir que as ordens de produção serão cumpridas. Assim, relata-se sobre o material em processamento, estado atual de cada ordem de produção, quantidades produzidas, utilização de equipamentos, etc. (MOREIRA, 2006). O controle da produção tem como objetivo acompanhar a produção, tomando informações para subsidiar correções necessárias. O controle pode assumir diversas formas, como verificadores de quantidades fabricadas, de qualidade e de custos (ERDMANN, 2007).

O desempenho operacional é a categoria de análise em organizações complexas que é o resultado da busca pelo aumento da produtividade, qualidade, inovação e lucratividade (SCHULZ, 2008).

O desenvolvimento de novos produtos é a categoria de análise em organizações complexas que é parte da estratégia empresarial. A estratégia pode ser product-out (desenvolve novos produtos com base na tecnologia que possui) ou market-in (desenvolvimento de novos produtos baseado em pesquisas de mercado) (MARTINS; LAUGENI, 2006).

A gestão ambiental é a categoria de análise em organizações complexas que compreende as diretrizes e princípios norteadores do planejamento e gerenciamento ambiental (SCHULZ, 2008). Para Valle (2002), a gestão ambiental engloba a definição de um 
conjunto de medidas e procedimentos que permitem controlar e reduzir os impactos que a organização exerce sobre o meio ambiente.

Instalações é a categoria de boa prática de análise em organizações complexas que propõe uma adaptação para organizações de serviço da categoria fábrica. Schulz (2008) afirma que a organização e a acessibilidade do ambiente de trabalho são aspectos fundamentais na gestão da produção, capazes de proporcionar desempenho mais ágil e eficaz por meio da sinergia criada entre as partes.

Schulz (2008) classifica a categoria de análise em organizações complexas: investimentos, como toda capitalização aplicada aos meios produtivos da organização. Silveira (2010) ressalta que investimentos em recursos humanos, tecnologia, equipamentos e qualidade produzem resultados diretos no desempenho operacional.

Schulz (2008) caracteriza a categoria de análise em organizações complexas, organização e cultura, como a identidade da empresa. Rivera (1995) afirma que as organizações são reguladas sistematicamente por regras fáticas e legais que são construídas, disseminadas e vivenciadas por todos os atores envolvidos. Essas regras determinam a influência dos atores, ou seja, o poder que cada um exerce dentro da organização.

O planejamento da produção é a categoria de análise em organizações complexas que corresponde ao conjunto de procedimentos nos quais o gerenciamento da produção se apoia para definir seus parâmetros, preparar e organizar as informações que irão permitir a programação e o controle da produção (ERDMANN, 2007).

A programação da produção é a categoria de análise em organizações complexas que corresponde ao ato de estabelecer antecipadamente as atividades da produção. Partindo dos dados estabelecidos no planejamento, a programação projeta o produto, roteiriza a produção e orienta quanto às quantidades a serem produzidas (ERDMANN, 2007).

A qualidade é a categoria de análise em organizações complexas que para Stevenson (2001) consiste na capacidade que determinado bem ou serviço tem de atender, ou mesmo superar as expectativas do cliente de maneira consistente.

Saúde e Segurança é a categoria de análise em organizações complexas que corresponde a um conjunto de ações organizacionais com objetivo de propiciar condições plenas de desenvolvimento humano no trabalho (SCHULZ, 2008). Esta categoria engloba aspectos relacionados à qualidade de vida no trabalho e saúde ocupacional (SILVEIRA, 2010).

A tecnologia é a categoria de análise em organizações complexas que está aliada à gestão das informações e processos. As organizações utilizam a tecnologia em processadores de texto ou equipamentos que facilitam ou automatizam algum processo (SLACK et al, 1999). Ao aliar-se à qualidade e melhorar o desempenho operacional, a tecnologia pode oferecer vantagens competitivas (SCHULZ, 2008).

Tempo de ciclo é a categoria de análise em organizações complexas que corresponde ao tempo total necessário para a conclusão de todo o processo produtivo. Slack et al (1999) afirmam que ao reduzir o tempo entre o consumidor solicitar o produto e recebê-lo, atinge-se o objetivo rapidez, um dos cinco objetivos de desempenho do setor produtivo. Entretanto, esta redução não pode acarretar em prejuízo em outros objetivos de desempenho, como redução de qualidade e confiabilidade, ou aumento excessivo de custos.

Para avaliar o desempenho organizacional a partir destas treze categorias é importante conhecer os principais fatores, observados nas boas práticas, que interferem no desempenho desta, ou seja, componentes críticos que afetam toda a cadeia da produção. Uma leitura a respeito de cada categoria (SILVEIRA, 2010; GONÇALVES; PIANA; ERDMANN, 2011) permitiu identificar pelo menos um componente variável que, de acordo com os preceitos do bom desempenho, da produtividade, do custo, da rapidez, da flexibilidade e da confiabilidade, inferem um melhor resultado para o sistema de produção, consequentemente, para a organização.

Os fatores de resultado são características muito importantes e vitais para a organização ser bem-sucedida no longo prazo e, portanto, altamente desejáveis (SLACK, et al, 1999). Ou ainda, são características capazes de proporcionar competitividade de forma direta à organização (GONÇALVES; PIANA; ERDMANN, 2011). São eles: confiabilidade, custos, rapidez, flexibilidade e qualidade.

Confiabilidade significa produzir e entregar bens e/ou serviços, em tempo hábil e nos prazos prometidos, comunicar as datas com clareza ao cliente, fazer a entrega pontualmente. Este conceito se estende às relações entre clientes internos.

O fator custos está relacionado com a capacidade de produzir bens e serviços, a custos mais baixos do que os concorrentes conseguem administrar. Mesmo as empresas que concorrem em outros aspectos que não preço estarão interessadas em manter seus custos baixos. 
Rapidez significa o tempo que o cliente deve esperar desde a emissão do pedido até o recebimento efetivo do produto. É o quão rápido a organização reage às demandas.

Flexibilidade está relacionada com a capacidade de atender a mudanças em bens e/ou serviços, prazos de entrega, volumes de produção, ampliação ou redução da variedade de bens ou serviços, aptidão a mudanças quando for necessário e com rapidez suficiente.

Qualidade significa fazer as coisas certas, entregar bens ou serviços conforme as especificações ou necessidades dos clientes, fazer produtos que realmente os clientes desejam sem cometer erros e de boa qualidade.

Em síntese o modelo de Silveira (2010) propõe que a avaliação organizacional contemple treze categorias de análise e considera ainda os cinco fatores de resultado, modelo este utilizado como referência para a elaboração da análise dos dados. A seguir apresenta-se a metodologia utilizada para que a pesquisa pudesse ser realizada.

\section{METODOLOGIA}

O objetivo deste artigo consiste em como um modelo de avaliação global pode gerar práticas de gestão institucional. Para isto utilizou-se como base quatro modelos de avaliação de desempenho: Avaliação institucional como sendo as avaliações realizadas pelo MEC (1) Avaliação institucional externa do MEC, (2) Avaliação de curso do MEC, (3) Avaliação de programa de pós-graduação CAPES, (4) Avaliação baseada em fatores de competitividade. Ao final destes quatro modelos se elaborará um modelo de avaliação global.

A pesquisa quanto aos fins pode ser considerada descritiva. É descritiva, pois descreve as informações obtidas por meio da pesquisa documental. Para Churchill (1987), pesquisa descritiva objetiva conhecer e interpretar a realidade sem nela interferir para modificá-la. A pesquisa descritiva expõe as características de determinada população ou de determinado fenômeno, mas não tem o compromisso de explicar os fenômenos que descreve, embora sirva de base para tal explicação. O método descritivo delineia o que se pesquisa, abordando quatro aspectos: investigação, registro, análise e interpretação dos fatos ocorridos no passado, para, através de generalizações, compreender o presente e o compromisso com o futuro.

Quanto a estratégia de pesquisa se utilizou a pesquisa bibliográfica e documental. Este tipo de pesqui- sa explica um problema a partir de referenciais teóricos publicados e documentos institucionais de livre acesso. Assim, busca-se conhecer e analisar as contribuições científicas sobre avaliação de desempenho (TRIVIÑOS, 1994). Nesse sentido, trabalhou-se com dados secundários.

A análise foi feita comparando os quatro modelos apresentados na fundamentação teórica deste artigo. Para a comparação entre os indicadores do modelo utilizou-se os princípios de análise da grounded theory. Assim, procedeu-se a codificação aberta, a codificação axial e a codificação seletiva. Desta forma, na codificação aberta se identificou os conceitos dos indicadores pertencentes aos quatro modelos. Na codificação axial se agrupou os indicadores em tornos dos subsistemas produtivos de acordo com a sua semelhança conceitual. Por fim, na codificação seletiva, se atinge a categorização final e apresenta-se o agrupamento definitivo.

\section{ANÁLISE DOS DADOS}

$\mathrm{Na}$ análise dos dados, as informações estão agrupadas em quatro categorias: foco de aplicação; metodologia de aplicação; resultados encontrados pelos métodos; e dimensões analisadas.

Ao comparar o foco da aplicação, é possível verificar que o modelo de avaliação baseada em fatores de competitividade é aplicado em sistemas de produção, enquanto os modelos do MEC e CAPES tem aplicação específica para IES, Cursos de graduação, Curso de Pós-Graduação Stricto Sensu.

Ao comparar metodologia de aplicação o modelo de avaliação baseada em fatores de competitividade trabalha com grupo focal e observação. Tem caráter qualitativo e trabalha com cenários qualitativos. Já os modelos elaborados pelo MEC e CAPES utilizam-se de análise documental e entrevistas. Possuem caráter quantitativo e possui cenários predominantemente quantitativos.

Comparando os resultados encontrados, o modelo de avaliação baseada em fatores de competitividade verifica relações entre as categorias de análise com os fatores de prática e tem por objetivo analisar o sistema de produção e verificar pontos de melhoria. Já os modelos do MEC e CAPES verificam a existência ou não de fatores específicos dentro das dimensões.

O quadro 1 comprara categorias de análise do modelo de avaliação baseada em fatores de competitividade com as dimensões da avaliação institucional, de curso e pós-graduação. 
QUADRO 1: COMPARAÇÃO DAS DIMENSÕES DOS MODELOS.

\begin{tabular}{|c|c|c|c|}
\hline $\begin{array}{l}\text { MODELO DE AVALIAÇÃO } \\
\text { BASEADA EM FATORES } \\
\text { DE COMPETITIVIDADE }\end{array}$ & INSTITUIÇÃO (MEC) & $\begin{array}{c}\text { CURSO } \\
\text { GRADUAÇÃO (MEC) }\end{array}$ & $\begin{array}{l}\text { PÓS-GRADUAÇÃO } \\
\text { (CAPES) }\end{array}$ \\
\hline $\begin{array}{l}\text { Controle da } \\
\text { Produção }\end{array}$ & $\begin{array}{l}\text { Planejamento e avaliação, especialmente } \\
\text { em relação aos processos, resultados e } \\
\text { eficácia da auto-avaliação institucional. }\end{array}$ & $\begin{array}{l}\text { Organização didático- } \\
\text { pedagógica; }\end{array}$ & Produção intelectual \\
\hline $\begin{array}{l}\text { Desempenho } \\
\text { Operacional }\end{array}$ & $\begin{array}{l}\text { Sustentabilidade financeira, tendo em } \\
\text { vista o significado social da continuidade } \\
\text { dos compromissos na oferta da educação } \\
\text { superior. }\end{array}$ & Infraestrutura. & $\begin{array}{l}\text { Corpo discente, } \\
\text { publicações,teses e } \\
\text { dissertações. Tempo de } \\
\text { formação de mestres e } \\
\text { doutores. }\end{array}$ \\
\hline Tecnologia & $\begin{array}{l}\text { Infra-estrutura física, especialmente a de } \\
\text { ensino e de pesquisa, biblioteca, recursos } \\
\text { de informação e comunicação. }\end{array}$ & $\begin{array}{l}\text { Organização didático- } \\
\text { pedagógica; }\end{array}$ & $\begin{array}{l}\text { Informações disponíveis } \\
\text { sobre o Programa. }\end{array}$ \\
\hline Instalações & $\begin{array}{l}\text { Infra-estrutura física, especialmente a de } \\
\text { ensino e de pesquisa, biblioteca, recursos } \\
\text { de informação e comunicação. }\end{array}$ & $\begin{array}{l}\text { Corpo docente e } \\
\text { tutorial }\end{array}$ & Infra-estrutura \\
\hline Gestão Ambiental & $\begin{array}{l}\text { A responsabilidade social da instituição, } \\
\text { considerada especialmente no que se } \\
\text { refere à sua contribuição em relação } \\
\text { à inclusão social, ao desenvolvimento } \\
\text { econômico e social, à defesa do meio } \\
\text { ambiente, da memória cultural, da } \\
\text { produção artística e do patrimônio } \\
\text { cultural. }\end{array}$ & $\begin{array}{l}\text { Organização didático- } \\
\text { pedagógica }\end{array}$ & - \\
\hline Investimentos & $\begin{array}{l}\text { Sustentabilidade financeira, tendo em } \\
\text { vista o significado social da continuidade } \\
\text { dos compromissos na oferta da educação } \\
\text { superior. }\end{array}$ & Infraestrutura & Corpo docente \\
\hline $\begin{array}{l}\text { Organização e } \\
\text { Cultura }\end{array}$ & $\begin{array}{l}\text { A comunicação com a sociedade; } \\
\text { As políticas de pessoal, de carreiras } \\
\text { do corpo docente e corpo técnico } \\
\text { administrativo, seu aperfeiçoamento, } \\
\text { seu desenvolvimento profissional e suas } \\
\text { condições de trabalho }\end{array}$ & - & $\begin{array}{l}\text { Corpo docente } \\
\text { Inserção e impacto do } \\
\text { programa. }\end{array}$ \\
\hline $\begin{array}{l}\text { Planejamento da } \\
\text { Produção }\end{array}$ & $\begin{array}{l}\text { A Missão e o Plano de Desenvolvimento } \\
\text { Institucional (PDI); } \\
\text { Organização e gestão da instituição, } \\
\text { especialmente o funcionamento e } \\
\text { representatividade dos colegiados, sua } \\
\text { independência e autonomia na relação } \\
\text { com a mantenedora, e a participação dos } \\
\text { segmentos da comunidade universitária } \\
\text { nos processos decisórios }\end{array}$ & Infraestrutura & Transparência. \\
\hline $\begin{array}{l}\text { Programação da } \\
\text { Produção }\end{array}$ & $\begin{array}{l}\text { A política para o ensino (graduação e pós- } \\
\text { graduação), a pesquisa, a extensão e as } \\
\text { respectivas normas de operacionalização, } \\
\text { incluídos os procedimentos para estímulo } \\
\text { à produção acadêmica, para as bolsas } \\
\text { de pesquisa, de monitoria e demais } \\
\text { modalidades. }\end{array}$ & $\begin{array}{l}\text { Organização didático- } \\
\text { pedagógica; } \\
\text { Corpo docente e } \\
\text { tutorial }\end{array}$ & $\begin{array}{l}\text { Corpo discente, teses e } \\
\text { dissertações }\end{array}$ \\
\hline
\end{tabular}




\begin{tabular}{|c|l|l|l|}
\hline $\begin{array}{c}\text { MODELO DE AVALIAÇÃO } \\
\text { BASEADA EM FATORES } \\
\text { DE COMPETITIVIDADE }\end{array}$ & \multicolumn{1}{|c|}{ INSTITUIÇÃO (MEC) } & $\begin{array}{c}\text { CURSO } \\
\text { GRADUAÇÃO (MEC) }\end{array}$ & $\begin{array}{c}\text { PÓS-GRADUAÇÃO } \\
\text { (CAPES) }\end{array}$ \\
\hline Saúde e Segurança & - & Infraestrutura \\
Qualidade & $\begin{array}{l}\text { Organização e gestão da instituição, } \\
\text { especialmente o funcionamento e } \\
\text { representatividade dos colegiados, sua } \\
\text { independência e autonomia na relação } \\
\text { com a mantenedora, e a participação dos } \\
\text { segmentos da comunidade universitária } \\
\text { nos processos decisórios }\end{array}$ & $\begin{array}{l}\text { Infraestrutura } \\
\text { Políticas de atendimento aos discentes. }\end{array}$ & $\begin{array}{l}\text { Corpo discente, teses e } \\
\text { dissertações } \\
\text { Corpo discente, teses e } \\
\text { dissertações } \\
\text { Qualidade das Teses e } \\
\text { Dissertações. } \\
\text { Publicações. }\end{array}$ \\
$\begin{array}{l}\text { Tempo de Ciclo } \\
\text { Desenvolvimento de } \\
\text { Novos Produtos }\end{array}$ & - & - & $\begin{array}{l}\text { Teses e dissertações } \\
\text { Linos legais e }\end{array}$ \\
\hline
\end{tabular}

Fonte: Elaborado pelos autores, 2015.

O quadro comparativo foi elaborado analisando os indicadores constantes nos relatórios de avaliação institucional e do curso, do MEC, e da pós-graduação, da CAPES, frente aos indicadores constantes no modelo de avaliação baseada em fatores de competitividade. Assim, quando existia congruência conceitual entre os indicadores, foi descrito que as perspectivas na qual elas pertencem teriam semelhança.

$O$ resultado da comparação entre os modelos será apresentado a seguir. O modelo de avaliação baseada em fatores de competitividade foi a base da junção para os demais modelos, que foram sendo incorporados, por meio de seus indicadores. Assim, tem-se como base um modelo genérico de avaliação de um sistema produtivo, acrescido de indicadores específicos de avaliação institucional, gerando um modelo global para gestão de IES. A base foi o modelo de avaliação baseada em fatores de competitividade porque este já indica para a geração de práticas administrativas.

$\mathrm{Na}$ sequência serão apresentadas as treze dimensões do modelo de avaliação baseada em fatores de competitividade e os indicadores específicos de avaliação institucional indexados a eles.

Assim, na dimensão controle da produção foram inseridos os indicadores ao modelo de avaliação baseada em fatores de competitividade.

\section{QUADRO 2: DIMENSÃO CONTROLE DA PRODUÇÃO.}

\begin{tabular}{|c|c|c|}
\hline CAPES & CURSO & INSTITUCIONAL \\
\hline $\begin{array}{l}\text { 1. } 80 \% \text { de } \\
\text { adequação do } \\
\text { NDP em relação } \\
\text { à Proposta do } \\
\text { Programa }\end{array}$ & $\begin{array}{l}\text { 1. Estrutura curricular prevista/ implantada contempla, } \\
\text { de maneira excelente, em uma análise sistêmica e } \\
\text { global; } \\
\text { 2. Quando o sistema de controle de produção e } \\
\text { distribuição de material didático previsto/implantado é } \\
\text { excelente para atender à demanda real; } \\
\text { 3. Quando os procedimentos de avaliação previstos/ } \\
\text { implantados utilizados nos processos de ensino- } \\
\text { aprendizagem atendem, de maneira excelente, à } \\
\text { concepção do curso. }\end{array}$ & $\begin{array}{l}\text { 1. Quando o planejamento e a avaliação, } \\
\text { especialmente em relação aos processos, } \\
\text { resultados e eficácia da auto-avaliação } \\
\text { institucional da IES estão coerentes com } \\
\text { o especificado no PDI; } \\
\text { 2. Quando a Comissão Própria de } \\
\text { Avaliação* está implantada e funciona } \\
\text { adequadamente, há efetiva participação } \\
\text { da comunidade interna. }\end{array}$ \\
\hline
\end{tabular}

Fonte: Elaborado pelos autores, 2015. 
O controle da produção é a categoria de análise que visa garantir que as ordens de produção serão cumpridas, tem como objetivo acompanhar a produção, tomando informações para subsidiar correções necessárias. $\mathrm{O}$ controle pode assumir diversas for- mas, como verificadores de quantidades fabricadas, de qualidade e de custos.

$\mathrm{Na}$ dimensão desempenho operacional foram inseridos os indicadores ao modelo de avaliação baseada em fatores de competitividade.

QUADRO 3: DIMENSÃO DESEMPENHO OPERACIONAL.

\begin{tabular}{|l|l|l|}
\hline CAPES & CURSO & INSTITUCIONAL \\
\hline $\begin{array}{l}\text { 1. Publicações qualificadas do } \\
\text { Programa por docente permanente } \\
\text { de } 150 \text { pontos Qualis; }\end{array}$ & $\begin{array}{l}\text { 1. Quando as ações acadêmico- } \\
\text { administrativas, em decorrência das } \\
\text { autoavaliações e das avaliações externas } \\
\text { (avaliação de curso, ENADE, CPC e } \\
\text { outras), no âmbito do curso, estão previstas/ } \\
\text { implantadas de maneira excelente; }\end{array}$ & $\begin{array}{l}\text { 1. Quando a sustentabilidade } \\
\text { financeira da IES está } \\
\text { coerente com a especificada } \\
\text { no PDI. }\end{array}$ \\
$\begin{array}{l}\text { 3. Tempo docenão de } 150 \text { pontos ou mais. } \\
\text { doutores }\end{array}$ & $\begin{array}{l}\text { 2. Quando pelo menos } 50 \% \text { dos docentes têm } \\
\text { mais de } 9 \text { produções nos últimos } 3 \text { anos. }\end{array}$ & \\
\hline
\end{tabular}

Fonte: Elaborado pelos autores, 2015.

O desempenho operacional é a categoria de análise em organizações complexas que identifica o resultado por meio da busca pelo aumento da produtividade, qualidade, inovação e lucratividade. Observa-se que nos três modelos adotados pelo MEC têm-se in- dicadores que promovem o desempenho operacional enquanto fator de competitividade.

$\mathrm{Na}$ dimensão equipamentos e tecnologia foram inseridos os indicadores ao modelo de avaliação baseada em fatores de competitividade.

QUADRO 4: DIMENSÃO EQUIPAMENTOS E TECNOLOGIA.

\begin{tabular}{|c|c|c|}
\hline CAPES & CURSO & INSTITUCIONAL \\
\hline $\begin{array}{l}\text { 1. Acesso as dissertações e teses. } \\
2 \text { Informações disponíveis sobre o } \\
\text { Programa. }\end{array}$ & $\begin{array}{l}\text { 1. Quando as tecnologias de informação e } \\
\text { comunicação (TICs) previstas/implantadas no } \\
\text { processo de ensino-aprendizagem permitem } \\
\text { executar, de maneira excelente, o projeto } \\
\text { pedagógico do curso. }\end{array}$ & $\begin{array}{l}\text { 1. Quando podem ser } \\
\text { verificadas ações adequadas } \\
\text { de atualização e ampliação } \\
\text { do acervo bibliográfico e dos } \\
\text { serviços da(s) biblioteca* (s). }\end{array}$ \\
\hline
\end{tabular}

Fonte: Elaborado pelos autores, 2015.

Essa categoria de análise em organizações complexas está aliada à gestão das informações e processos. As organizações utilizam a tecnologia em processadores de texto ou equipamentos que facilitam ou automatizam al- gum processo, gerando melhorias de desempenho.

$\mathrm{Na}$ dimensão instalações foram inseridos os indicadores ao modelo de avaliação baseada em fatores de competitividade. 
QUADRO 5: DIMENSÃO INSTALAÇÃO.

\begin{tabular}{|l|l|l|}
\hline CAPES & CURSO & INSTITUCIONAL \\
\hline $\begin{array}{l}\text { 1. Infra-estrutura para ensino, } \\
\text { pesquisa e extensão. }\end{array}$ & $\begin{array}{l}\text { 1. Quando os gabinetes de trabalho } \\
\text { implantados para os docentes em tempo } \\
\text { integral, os espaços as atividades de } \\
\text { coordenação, sala de aula e sala dos } \\
\text { professores são excelentes considerando, em } \\
\text { uma análise sistêmica e global. }\end{array}$ & $\begin{array}{l}\text { 1. Quando a infra-estrutura } \\
\text { física da IES, especialmente } \\
\text { a de ensino e pesquisa, } \\
\text { biblioteca, recursos de } \\
\text { informação e comunicação, } \\
\text { está coerente com a } \\
\text { especificada no PDI. }\end{array}$ \\
\hline
\end{tabular}

Fonte: Elaborado pelos autores, 2015.

Essa é a categoria de boa prática de análise que propõe uma adaptação para organizações de serviço da categoria ambiente interno. Está relacionada à infraestrutura física e operacional. Observa-se que nos três modelos adotados pelo MEC têm-se indicadores que promovem a instalação física enquanto fator de competitividade.

Na dimensão gestão ambiental foram inseridos os indicadores ao modelo de avaliação baseada em fatores de competitividade.

QUADRO 6: DIMENSÃO GESTÃO AMBIENTAL.

\begin{tabular}{|l|l|l|}
\hline CAPES & CURSO & INSTITUCIONAL \\
\hline Nenhum & Nenhum & $\begin{array}{l}\text { 1. Quando as ações da IES com vistas à defesa do meio ambiente, } \\
\text { do patrimônio cultural e da produção artística resultam de diretrizes } \\
\text { institucionais e estão adequadamente implantadas e acompanhadas. }\end{array}$ \\
\hline
\end{tabular}

Fonte: Elaborado pelos autores, 2015.

A gestão ambiental compreende as diretrizes e princípios norteadores do planejamento e gerenciamento ambiental, englobando a definição de um conjunto de medidas e procedimentos que permitem controlar e reduzir os impactos que a organização exerce sobre o meio ambiente. Observa-se no quadro apresentado anteriormente que a dimensão gestão ambiental apresenta um indicador de desempenho, indicando a falta da análise desta dimensão nos modelos adotados pelo MEC.

Na dimensão investimento foram inseridos os indicadores ao modelo de avaliação baseada em fatores de competitividade.

\section{QUADRO 7: DIMENSÃO INVESTIMENTO.}

\begin{tabular}{|l|l|l|}
\hline CAPES & \multicolumn{1}{|c|}{ CURSO } & \multicolumn{1}{c|}{ INSTITUCIONAL } \\
\hline $\begin{array}{l}\text { 1. } 70 \% \text { do NDP em relação ao } \\
\text { total de docentes do Programa } \\
\text { com distribuição correta da } \\
\text { carga horária. }\end{array}$ & $\begin{array}{l}\text { 1. Quando o acervo } \\
\text { da bibliografia } \\
\text { básica, com no } \\
\text { mínimo três títulos } \\
\text { por unidade } \\
\text { curricular }\end{array}$ & $\begin{array}{l}\text { 1. Quando se verifica a adequação entre a proposta de } \\
\text { desenvolvimento da IES, incluindo-se a captação de } \\
\text { recursos, e o orçamento previsto, a compatibilidade entre } \\
\text { cursos oferecidos e as verbas e os recursos disponíveis, e } \\
\text { existe controle entre as despesas efetivas e as referentes à } \\
\text { despesa correntes, de capital e de investimento; } \\
\text { programas. }\end{array}$ \\
$\begin{array}{l}\text { 3. Carga letiva entre os } \\
\text { docentes. }\end{array}$ & $\begin{array}{l}\text { 2. Quando existem políticas de aquisição de equipamentos } \\
\text { e de expansão e/ou conservação do espaço físico } \\
\text { necessárias à adequada implementação dos programas de } \\
\text { ensino, pesquisa e extensão. }\end{array}$ \\
\hline
\end{tabular}

Fonte: Elaborado pelos autores, 2015. 
Corresponde a capitalização aplicada a instituição, os investimentos em recursos humanos, tecnologia, equipamentos e qualidade produzem resultados diretos no desempenho operacional. Observa-se que nos três modelos adotados pelo MEC têm-se indica- dores que promovem o investimento enquanto fator de competitividade.

$\mathrm{Na}$ dimensão organização e cultura foram inseridos os indicadores ao modelo de avaliação baseada em fatores de competitividade.

QUADRO 8: DIMENSÃO ORGANIZAÇÃO E CULTURA.

\begin{tabular}{|c|c|c|}
\hline CAPES & CURSO & INSTITUCIONAL \\
\hline $\begin{array}{l}\text { 1. Formação } \\
\text { Docente } \\
\text { diversificada. } \\
\text { 2. Inserção e } \\
\text { impacto. } \\
\text { 3. Transparência. }\end{array}$ & $\begin{array}{l}\text { 1. Quando o perfil profissional expressa, de maneira } \\
\text { excelente, as competências do egresso; } \\
\text { 2. Quando o percentual dos docentes do curso com } \\
\text { titulação obtida em programas de pós-graduação } \\
\text { stricto sensu é maior ou igual a } 75 \% \text {. } \\
\text { 3. Quando o percentual do corpo docente previsto/ } \\
\text { efetivo com regime de trabalho de tempo parcial ou } \\
\text { integral é maior ou igual que } 80 \% \text {. }\end{array}$ & $\begin{array}{l}\text { 1. Quando os canais de comunicação } \\
\text { e sistemas de informação para a } \\
\text { interação interna e externa funcionam } \\
\text { adequadamente, são acessíveis às } \\
\text { comunidades interna e externa e } \\
\text { possibilitam a divulgação das ações da } \\
\text { IES*; } \\
\text { 2. Formação docente e política de } \\
\text { pessoal adequada ao PDI; } \\
\text { 3. Capacitação docente. }\end{array}$ \\
\hline
\end{tabular}

Fonte: Elaborado pelos autores, 2015.

Corresponde a identidade da empresa, são regras fáticas e legais que são construídas, disseminadas e vivenciadas por todos os membros da organização. Essas regras determinam o poder que cada um exerce dentro da organização.

Na dimensão planejamento da produção foram inseridos os indicadores ao modelo de avaliação baseada em fatores de competitividade.

\section{QUADRO 9: DIMENSÃO PLANEJAMENTO DA PRODUÇÃO.}

\begin{tabular}{|l|l|l|}
\hline CAPES & CURSO & INSTITUCIONAL \\
\hline $\begin{array}{l}\text { 1. máximo de } \\
\text { oito orientandos } \\
\text { por docente } \\
\text { permanente. }\end{array}$ & $\begin{array}{l}\text { 1. PDI estão previstas/implantadas, de maneira } \\
\text { excelente, no âmbito do curso; } \\
\text { 2. Quando o número de vagas previstas/implantadas } \\
\text { corresponde, de maneira excelente, à dimensão do } \\
\text { corpo docente e às condições de infraestrutura da IES; } \\
\begin{array}{l}\text { 3. Quando a média entre o número de docentes } \\
\text { do curso (equivalentes 40h) e o número de vagas } \\
\text { previstas/implantadas é de 1 docente para 130. }\end{array}\end{array}$ & $\begin{array}{l}\text { 1. Implementação do PDI e Articulação } \\
\text { entre o PDI* e os processos de avaliação } \\
\text { institucional (auto-avaliação e } \\
\text { avaliaçães externas); }\end{array}$ \\
2. Funcionamento dos Conselhos \\
Superiores e Colegiados.
\end{tabular}

Fonte: Elaborado pelos autores, 2015.

O planejamento da produção é a categoria de análise que corresponde ao conjunto de procedimentos nos quais o gerenciamento da produção se apoia para definir seus parâmetros, preparar e organizar as in- formações.

$\mathrm{Na}$ dimensão programação da produção foram inseridos os indicadores ao modelo de avaliação baseada em fatores de competitividade. 
QUADRO 10: DIMENSÃO PROGRAMAÇÃO DA PRODUÇÃO.

\begin{tabular}{|c|c|c|}
\hline CAPES & CURSO & INSTITUCIONAL \\
\hline $\begin{array}{l}\text { 1. Percentual de titulados no ano- } \\
\text { base considerando-se o número de } \\
\text { discentes no final do ano-anterior: } \\
\text { Proporção Mestrado } 40 \% \text { ou mais; } \\
\text { Proporção Doutorado } 25 \% \text { ou mais. }\end{array}$ & $\begin{array}{l}\text { 1. PPC contempla, de maneira excelente, as } \\
\text { demandas efetivas de natureza econômica e } \\
\text { social. } \\
\text { 2. Atuação do NDE e coordenador do curso. }\end{array}$ & $\begin{array}{l}\text { 1. Políticas institucionais } \\
\text { para graduação, pós- } \\
\text { graduação, pesquisa e } \\
\text { extensão. }\end{array}$ \\
\hline
\end{tabular}

Fonte: Elaborado pelos autores, 2015.

É a categoria de análise que corresponde ao ato de estabelecer antecipadamente as atividades do curso. Partindo dos dados estabelecidos no planejamento, a programação projeta o curso. Observa-se que nos três modelos adotados pelo MEC têm-se indicadores que promovem a programação da produção enquanto fator de competitividade.

Na dimensão saúde e segurança foram inseridos os indicadores ao modelo de avaliação baseada em fatores de competitividade.

QUADRO 11: DIMENSÃO SAÚDE E SEGURANÇA.

\begin{tabular}{|l|l|l|}
\hline CAPES & CURSO & INSTITUCIONAL \\
\hline Nenhum & $\begin{array}{l}\text { 1. Quando existe o comitê de ética } \\
\text { funcionando de maneira excelente e } \\
\text { homologado pela CONEP. }\end{array}$ & Nenhum \\
\hline
\end{tabular}

Fonte: Elaborado pelos autores, 2015.

Saúde e Segurança é a categoria de análise que corresponde a um conjunto de ações organizacionais com objetivo de propiciar condições plenas de desenvolvimento humano no trabalho, relaciona-se à qualidade de vida no trabalho e saúde ocupacional. Observa-se no quadro apresentado anteriormente que a dimensão saúde e segurança apresenta um indicador de desempenho, indicando a falta da análise desta dimensão nos modelos adotados pelo MEC.

$\mathrm{Na}$ dimensão qualidade foram inseridos os indicadores ao modelo de avaliação baseada em fatores de competitividade.

QUADRO 12: DIMENSÃO QUALIDADE.

\begin{tabular}{|c|c|c|}
\hline CAPES & CURSO & INSTITUCIONAL \\
\hline $\begin{array}{l}\text { 1. } 80 \% \text { ou mais das teses e } 50 \% \\
\text { ou mais das dissertações gerarem } \\
\text { publicações referenciadas no Qualis; } \\
\text { 2. Relações internacionais com } \\
\text { outras IES. }\end{array}$ & $\begin{array}{l}\text { 1. Quando os laboratórios especializados } \\
\text { implantados com respectivas normas de } \\
\text { funcionamento, utilização e segurança } \\
\text { atendem, de maneira excelente, em uma } \\
\text { análise sistêmica e global. }\end{array}$ & $\begin{array}{l}\text { 1. Quando a gestão } \\
\text { institucional se pauta em } \\
\text { princípios de qualidade, } \\
\text { e resulta de diretrizes de } \\
\text { ações. }\end{array}$ \\
\hline
\end{tabular}

Fonte: Elaborado pelos autores, 2015.

A qualidade é a categoria de análise em organizações complexas que mede a capacidade que determinado bem ou serviço tem de atender, ou mesmo superar as expectativas do cliente de maneira consistente.

$\mathrm{Na}$ dimensão tempo de ciclo foram inseridos os indicadores ao modelo de avaliação baseada em fatores de competitividade. 
QUADRO 13: DIMENSÃO TEMPO DE CICLO.

\begin{tabular}{|l|l|l|}
\hline CAPES & CURSO & INSTITUCIONAL \\
\hline $\begin{array}{l}\text { 1. Tempo de } \\
\text { formação de } \\
\text { mestresem } 24 \text { meses } \\
\text { e doutores em } 48 \\
\text { meses. }\end{array}$ & $\begin{array}{l}\text { 1. Tempo mínimo } \\
\text { e máximo para } \\
\text { integralização do } \\
\text { curso. }\end{array}$ & $\begin{array}{l}\text { 1. Quando se verifica a adequação das políticas de acesso, seleção } \\
\text { e permanência de estudantes (critérios utilizados, acompanhamento } \\
\text { pedagógico, espaço de participação e de convivência) praticadas } \\
\text { pela IES e há adequada relação com as políticas públicas e com o } \\
\text { contexto social. }\end{array}$ \\
\hline
\end{tabular}

Fonte: Elaborado pelos autores, 2015.

Tempo de ciclo é a categoria de análise em organizações complexas que corresponde ao tempo total necessário para a conclusão de todo o processo produtivo. Observa-se que nos três modelos adotados pelo MEC têm-se indicadores que promovem a análise do tempo de ciclo enquanto fator de competitividade.
Observa-se que nos três modelos adotados pelo MEC têm-se indicadores que promovem o tempo de ciclo enquanto fator de competitividade.

Na dimensão desenvolvimento de novos produtos foram inseridos os indicadores ao modelo de avaliação baseada em fatores de competitividade.

QUADRO 14: DIMENSÃO DESENVOLVIMENTO DE NOVOS PRODUTOS.

\begin{tabular}{|l|l|l|}
\hline CAPES & CURSO & INSTITUCIONAL \\
\hline $\begin{array}{l}\text { 1. Participação dos docentes em } \\
\text { pesquisa e desenvolvimento de } \\
\text { projetos superior a } 70 \% .\end{array}$ & Nenhum & Nenhum \\
\hline
\end{tabular}

Fonte: Elaborado pelos autores, 2015.

Observa-se no quadro apresentado anteriormente que a dimensão de desenvolvimento de novos produtos apresenta um indicador de desempenho, indicando a falta da análise desta dimensão nos modelos adotados pelo MEC.

O resultado da comparação entre os modelos será apresentado a seguir. A modelo de avaliação baseada em fatores de competitividade foi a base da junção para os demais modelos, que foram sendo incorporados, por meio de seus indicadores. Assim, tem-se como base um modelo genérico de avaliação de um sistema produtivo, acrescido de indicadores específicos de avaliação institucional, gerando um modelo global para gestão de IES. A base foi o modelo de avaliação baseada em fatores de competitividade porque este já indica para a geração de práticas administrativas.

O modelo de avaliação de desempenho baseado em fatores de competitividade, conforme apresenta- do na fundamentação teórica deste artigo, apresenta a representatividade dos sistemas de produção dividida em treze categorias de análise (Quadros II ao XIV) e considera cinco fatores de resultado.

Duas informações de saídas são esperadas para este modelo global desenvolvido. Uma para os indicadores e outra para as dimensões.

$\mathrm{Na}$ saída de dados espera-se que sejam apresentados os indicadores destacados por cor. Assim, quando o indicador do modelo de avaliação baseada em fatores de competitividade apresentar nota um destaca-se com a cor vermelha. $\mathrm{O}$ indicador com nota dois com a cor vermelha claro. A cor alaranjada corresponderá a um indicador nota três. A cor amarela será apresentada juntamente com um indicador quatro. $\mathrm{O}$ indicador cinco será apresentado com a cor verde.

A seguir é apresentado um exemplo de aplicação do modelo gerado. 
FIGURA 1- EXEMPLO DE APLICAÇÃO DO MODELO GERADO.

\begin{tabular}{|c|c|c|c|c|c|c|c|c|}
\hline \multirow[b]{2}{*}{ Planejamento da Produção } & \multicolumn{2}{|r|}{ Custo } & \multicolumn{2}{|c|}{ Flexibilidade } & \multicolumn{2}{|c|}{ Qualidade } & \multicolumn{2}{|c|}{ Média } \\
\hline & $\sqrt{2}$ & 2 & 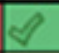 & 4 & 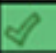 & 4 & & 3,3 \\
\hline Programação da Produção & 2 & 2 & 8 & 3 & $\vartheta$ & 4 & 8 & 3,0 \\
\hline Controle da Produção & $\infty$ & 4 & ? & 3 & $\bar{x}$ & 2 & ? & 3,0 \\
\hline Desempenho Operacional & 2 & 2 & 8 & 3 & 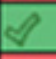 & 4 & 8 & 3,0 \\
\hline DNP & 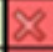 & 2 & 2 & 2 & $\underline{x}$ & 2 & 2 & 2,0 \\
\hline Instalações & $x^{3}$ & 2 & $x^{3}$ & 2 & 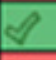 & 4 & 8 & 2,7 \\
\hline Gestão Ambiental & $\bar{x}$ & 2 & 8 & 3 & $\bar{x}$ & 2 & $x$ & 2,3 \\
\hline Investimentos & 2 & 2 & 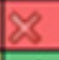 & 2 & $\underline{x}$ & 2 & 2 & 2,0 \\
\hline Organização e Cultura & 8 & 3 & 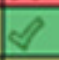 & 4 & $\bar{x}$ & 2 & 8 & 3,0 \\
\hline Qualidade & 8 & 3 & 8 & 3 & $\bar{x}$ & 2 & 8 & 2,7 \\
\hline Saúde e Segurança & $\bar{x}$ & 2 & 8 & 2 & 8 & 3 & $x$ & 2,3 \\
\hline Tecnologia & $\bar{x}$ & 2 & 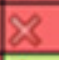 & 2 & 8 & 3 & $x$ & 2,3 \\
\hline Tempo de Ciclo. & 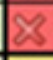 & 2 & 8 & 3 & $\sqrt{3}$ & 2 & $x$ & 2,3 \\
\hline Média & $x$ & 2,3 & 8 & 2,8 & 8 & 2,8 & $x$ & 2,6 \\
\hline
\end{tabular}

Fonte: Elaborado pelos autores, 2015.

Para as treze dimensões espera-se como saída de informação um gráfico do tipo radar com o cruzamento das informações das notas dos indicadores provenientes dos fatores de resultado.

\section{CONSIDERAÇÕES FINAIS}

O objetivo deste artigo consiste em como um modelo de avaliação global pode gerar práticas de gestão institucional. Após o exposto na análise dos dados considera-se que o objetivo do artigo foi alcançado. Isso porque utilizou-se como base quatro modelos de avaliação de desempenho: Avaliação institucional como sendo as avaliações realizadas pelo MEC: Avaliação institucional externa do MEC, Avaliação de curso do MEC, Avaliação de programa de pós-graduação CAPES, e Avaliação baseada em fatores de competitividade. O modelo de avaliação baseado em fatores de competitividade desenvolvido para organizações complexas apresenta treze categorias de análise: Controle da Produção, Desempenho Operacional, Desenvolvimento de Novos Produtos, Fábrica / Instalações, Gestão Ambiental, Investimentos, Organização e Cultura, Planejamento da Produção, Programação da Produção, Qualidade, Saúde e Segurança, Tecnologia, e Tempo de Ciclo.

O modelo de avaliação baseada em fatores de competitividade foi a base da junção para os demais modelos, que foram sendo incorporados, por meio de seus indicadores. Assim, tem-se como base um modelo genérico de avaliação de um sistema produtivo, acrescido de indica- dores específicos de avaliação institucional, gerando um modelo global para gestão de IES. A base foi o modelo de avaliação baseada em fatores de competitividade porque este já indica para a geração de práticas administrativas.

A construção do modelo de avaliação de desempenho global tem como base os modelos inicialmente apresentados e, respectivamente, fundamentados. Espera-se que o modelo de avaliação desenvolvido neste estudo traga reflexões e contribuições para a melhoria do desempenho das organizações educacionais e para o aprofundamento de estudos de novos modelos.

O modelo de avaliação de desempenho global desenvolvido para as instituições complexas pretende trazer a discussão uma forma de avaliar que integre os fatores que geram competitividades para as IES e identificar que modelos utilizados atualmente pelo Ministério da Educação não comporta todos estes fatores. Observa-se que três dimensões não são contempladas de forma satisfatória pelos modelos, a saber, o desenvolvimento de novos produtos, saúde e segurança e gestão ambiental.

A adoção de um novo modelo avaliativo gera mudança organizacional e aprendizado, desta forma a organização estará em nível evolutivo superior em termos de avaliação de desempenho organizacional, pois terá adquirido competências para lidar com estas mudanças.

Espera-se também que os resultados deste artigo sirvam como ponto inicial para o desenvolvimento de outras pesquisas. E, por fim, acredita-se que, com o aprofundamento dos estudos, será possível retroalimentar o processo de desenvolvimento científico. 
BRASIL. Ministério da Educação (MEC). Educação Superior: O que é o Conceito Preliminar de Curso? Disponível em: <http://portal.mec.gov.br/>. Acesso em 25 de Nov. 2012.

Sistema nacional de avaliação da educação superior (SINAES): da concepção à regulamentação. Disponível em: <http://www.inep.gov.br/download/catalogo_dinamico/provao/2004/sinaes.pdf>. Acessoem: 24 denov.2004.

CHURCHILL, G.A. Marketing research: methodological foundations. Chicago: The Dryden Press, 1987.

CRISPIM, S.; LUGOBONI, L. Avaliação de desempenho organizacional: Análise comparativa dos modelos teóricos e pesquisa de aplicação nas Instituições de Ensino Superior da Região Metropolitana de São Paulo. Rev. Portuguesa e Brasileira de Gestão, Lisboa, v. 11, n. 1, jan. 2012. Disponível em: < http://www.scielo.gpeari.mctes. pt/scielo.php?script=sci_arttext\&pid=S1645-44642012000100005\&lng=pt\&nrm=iso $>$. Acessos em 22 nov. 2013.

CONTO, S. M.; BRITTO, J. C.; CYRNE, C. C. da S.. Alinhamento das dez dimensões de avaliação do sinaes e o planejamento estratégico: construção de uma proposta para um centro universitário. In: Colóquio Internacional sobre Gestão Universitária na América do Sul, 11, Universidade Federal de Santa Catarina - UFSC, Florianópolis, 7-9 dez. 2011.

COORDENAÇÃO DE APERFEIÇOAMENTO DE PESSOAL DE NÍVEL SUPERIOR (CAPES). Avaliação da Pós-Graduação. Disponível em: http://www.capes.gov.br/avaliacao/avaliacao-da-pos-graduacao> Acesso em: 26 out. 2013.

DIAS SOBRINHO, J. Avaliação ética e política em função da educação como direito público ou como mercadoria. Avaliação, Campinas; Sorocaba, SP, v. 25, n. 88, p. 703-725, Especial, mar. 2004.

ENSSLIN, L. E.; et al. Modelo multicritério para avaliação e seleção de projetos de pesquisa e desenvolvimento em uma empresa distribuidora de energia. Revista Gestão Industrial. Universidade Tecnológica Federal do Paraná UTFPR, Ponta Grossa, v. 08, n. 01: p. 164-198, 2012.

ERDMANN, R.H. Administração da Produção: planejamento, programação e controle. Florianópolis: Editora Papa Livro, 2007.

GALVÃO, H. M.; CORRÊA, H. L.; ALVES, J. L. Modelo de avaliação de desempenho global para instituição de ensino superior. Rev. Adm. UFSM, Santa Maria, v. 4, n. 3, p. 425-441 set./dez. 2011

GONÇALVES. C.; PIANA, J.; ERDMANN, R. H. As avaliações de desempenho e uma nova proposta de método de diagnóstico e gestão organizacional. In: Enegep - XXXI Encontro Nacional de Engenharia de Produção, 2011, Belo Horizonte. XXXIEnegep. Rio de Janeiro: Abepro, 2011. v. 1. p. 1-8.

GRZEBIELUCKAS, Cleci et al. Instrumento para identificação das necessidades do consumidor no processo de desenvolvimento do design: um estudo ilustrado com o projeto de um automóvel. Gest. Prod., São Carlos, v. 18, n. 2, 2011 . Available from $<$ http://www.scielo.br/scielo.php?script=sci_arttext\&pid=S0104-530X2011000200009\&1 ng=en\&nrm=iso $>$. access on 20 Nov. 2013.

INEP. Censo da Educação Superior 2010. Brasília: Outubro de 2011.

LIMA, C.R.M; SOARES, T.C.; LIMA, M.A. Utilização do Balanced Scorecard em Instituições de Ensino Superior. RIC - Revista de Informação Contábil. Recife, v. 6, n. 3, p. 1-13, Jul-Set, 2012. 
LONGARAY, A.A.; ENSSLIN, L.. Uso da MCDA na identificação e mensuração da performance dos critérios para a certificação dos hospitais de ensino no âmbito do SUS. Prod., São Paulo, 2013. Available from <http://www. scielo.br/scielo.php?script=sci_arttext\&pid $=\mathrm{S} 0103-65132013005000021 \& \operatorname{lng}=\mathrm{en} \& \mathrm{nrm}=\mathrm{iso}>$. access on 22 Nov. 2013. Epub May 14, 2013.

LUHMANN, N. O conceito de sociedade. In: NEVES, C. B.; SAMIOS, E. M. B..NiklasLuhmann: a nova teoria dos sistemas. Porto Alegre: Ed. UFRGS, 1997

MACCARI, E. A.; LIMA, M. C.; RICCIO, E.L. Uso do sistema de avaliação da CAPES por programas de pósgraduação em administração no Brasil. Revista de Ciências da Administração, v. 11, n. 25, set/dez, 2009.

MARTINS, P.G.; LAUGENI, F.P. Administração da produção. São Paulo: Saraiva, 2006.

MEYER JR., V.; MEYER, B. Planejamento estratégico nas instituições de ensino superior: técnica ou arte? In: Colóquio Internacional sobre Gestão Universitária na América do Sul, 4, Universidade Federal de Santa Catarina - UFSC, Florianópolis, 8-10 dez. 2004.

MOREIRA, F. K. Diagnóstico de organizações complexas: o caso da unidade de pronto atendimento sul de Florianópolis. Dissertação (Mestrado em Administração)- Universidade Federal de Santa Catarina. Programa de pós-graduação em Administração. Florianópolis, 2011.

MOREIRA, D.A. Administração da Produção e Operações. São Paulo: Thompson Learning, 2006.

MORIN, E. Ciência com consciência. Lisboa: Europa-América, 1982.

PETERSON, M.. Avaliação institucional na educação de nível superior. In: SOUSA, E da C. B. Machado de (Org.). Avaliação Institucional. 2.ed. Brasília: Universidade de Brasília, IESB, 2000.

POLIDORI, M. M.; MARINHO-ARAUJO, C. M. M.; BARREYRO, G. B. SINAES: Perspectivas e desafios na avaliação da educação superior brasileira. Ensaio: Avaliação de Políticas Públicas Educacionais. Rio de Janeiro, v.14, n.53, p. 425-436, out./dez. 2006.

RISTOFF, D. I. Apresentação In DIAS SOBRINHO, José. Universidade e avaliação: entre a ética e o mercado. Florianópolis: Insular, 2002.

.; GIOLO, J. O Sinaes como sistema. R B P G, v. 3, n. 6, p. 193-213, 2006.

ROMAN, D. J.;PIANA, J.; LOZANO, D.; MELLOJ.;ERDMANN, R. H.. Fatores de competitividade organizacional. BBR, Vitória, v. 9, n. 1, Art. 2, p. 27-46, jan./mar. 2012.

.; OSINSKI, M.; ERDMANN, R. H..A substantive theory on the implementation process of operational performance improvement methods. RAUSP, v. 52, n. 2. April - June 2017.

RUBEN, B. D. Toward a balanced scorecard for higher education: rethinking the college and university excellence indicators framework. Higher Education Forum, Center for Organizational Development and Leadership, Rutgers University, fall, 1999.

SCHULZ, A. A. Relações complexas na administração da produção. Dissertação (Mestrado em Administração) - Universidade Federal de Santa Catarina. Programa de pós-graduação em Administração. Florianópolis, 2008.

SILVA, M. das G. M.. A produção docente e a avaliação dos programas de pós-graduação: um estudo na pós- 


\section{raUnP}

graduação da UFMT. Revista de Educação Pública, v. 18, n. 37, 2009.

SILVEIRA, A.M.O.L. Ferramenta de diagnóstico para organizações complexas. Dissertação (Mestrado em Administração)- Universidade Federal de Santa Catarina. Programa de pós-graduação em Administração. Florianópolis, 2010.

SLACK, N. et al. Administração da Produção. São Paulo: Atlas, 1999.

STEVENSON, W. J. Administração das Operações de Produção. 6 ed. Rio de Janeiro: LTC, 2001

TRIVIÑOS, A.N.S. Introdução à pesquisa em ciências sociais: a pesquisa qualitativa em educação. São Paulo: Atlas, 1994.

VALLE, C.E. Qualidade ambiental: ISO 14000. São Paulo: Editora SENAC, 2002. 